\title{
A Case of Successful Iliofemoral Vein Stenting from Great Saphenous Vein Access
}

\author{
Hae Won Jung, ${ }^{1}$ Chul-Min Ahn, ${ }^{2}$ Young-Guk Ko ${ }^{2}$
}

${ }^{1}$ Department of Cardiology, Daegu Catholic University Medical Center, Daegu, Republic of Korea; ${ }^{2}$ Division of Cardiology, Severance Cardiovascular Hospital, Yonsei University College of Medicine, Seodaemun-gu, Seoul, Republic of Korea

\section{ABSTRACT}

Chronic venous disease is strongly associated with morbidity and leads to considerable medical costs. Therefore, its clinical significance is very important. Currently, iliac vein stenting is the first treatment option for chronic venous disease due to iliac vein obstruction. For iliac vein stenting, ipsilateral femoral or popliteal vein access is common. However, great saphenous vein access may be a good alternative if there is obstruction in the ipsilateral femoropopliteal vein. Until now, there has been no reported case of successful iliac vein stenting using great saphenous vein access. We report the first successful case of iliofemoral vein stenting from great saphenous vein access.

\section{INTRODUCTION}

Endovenous stenting in patients with chronic iliofemoral vein obstruction is a proven therapy with its therapeutic efficacy and safety [Neglen 2007; Raju 2009b; Raju 2010; Razavi 2015; Wen-da 2016]. In general, a stent is inserted through ipsilateral femoral or popliteal vein access into the iliac vein lesion. This is the first case of successful iliofemoral vein stenting through ipsilateral great saphenous vein (GSV) access due to the occlusion of the ipsilateral femoropopliteal vein.

\section{CASE}

Six months ago, a 31-year-old man came to our hospital because his left leg edema had worsened after a traffic accident (Figure 1). He had a history of left leg muscle rupture and hematoma after leg stretching a year ago. Chronic phlebitis of left iliac vein and chronic deep vein thrombosis of the left femoropopliteal vein was a cause for concern on the CT scan. We performed embolectomy for left common femoral vein (LCFV), because a significant amount of thrombosis was a cause for concern on the CT scan. However, the LCFV was almost closed; only a small amount of blood clots was removed. He was discharged with rivaroxaban $15 \mathrm{mg} /$ day. After discharge, he continued complaining of his left leg swelling; at this time, we decided to treat the patient with endovascular treatment (EVT). A CT scan was

Received fuly 2, 2018; accepted October 3, 2018.

Correspondence: Chul-Min Ahn, Division of Cardiology, Severance Cardiovascular Hospital, Yonsei University College of Medicine, 50 Yonsei-ro, Seodaemun-gu, Seoul, 03722, Republic of Korea; (DRCELLO@yubs.ac). done once again; a severely thinned left iliac vein (LIV), left femoral vein (LFV), and left popliteal vein (LPV), and a huge left great saphenous vein (LGSV) were seen (Figures $2 \mathrm{~A}-2 \mathrm{C}$ ). In a $3 \mathrm{D}$ reconstruction image, we found a huge LGSV drained to the femoral vein at the saphenofemoral junction (SFJ) and collateral veins (Figure 3). Because of the thinning of the left femoral and popliteal veins, it was considered impossible to use these veins as an access. Therefore, we decided to approach the left iliofemoral vein lesion through the giant LGSV.

The patient was placed in the supine position for EVT. First, the retrograde approach through the right common femoral vein was done. A 0.018-inch guidewire with Rubicon support catheter (Boston Scientific Corporation,

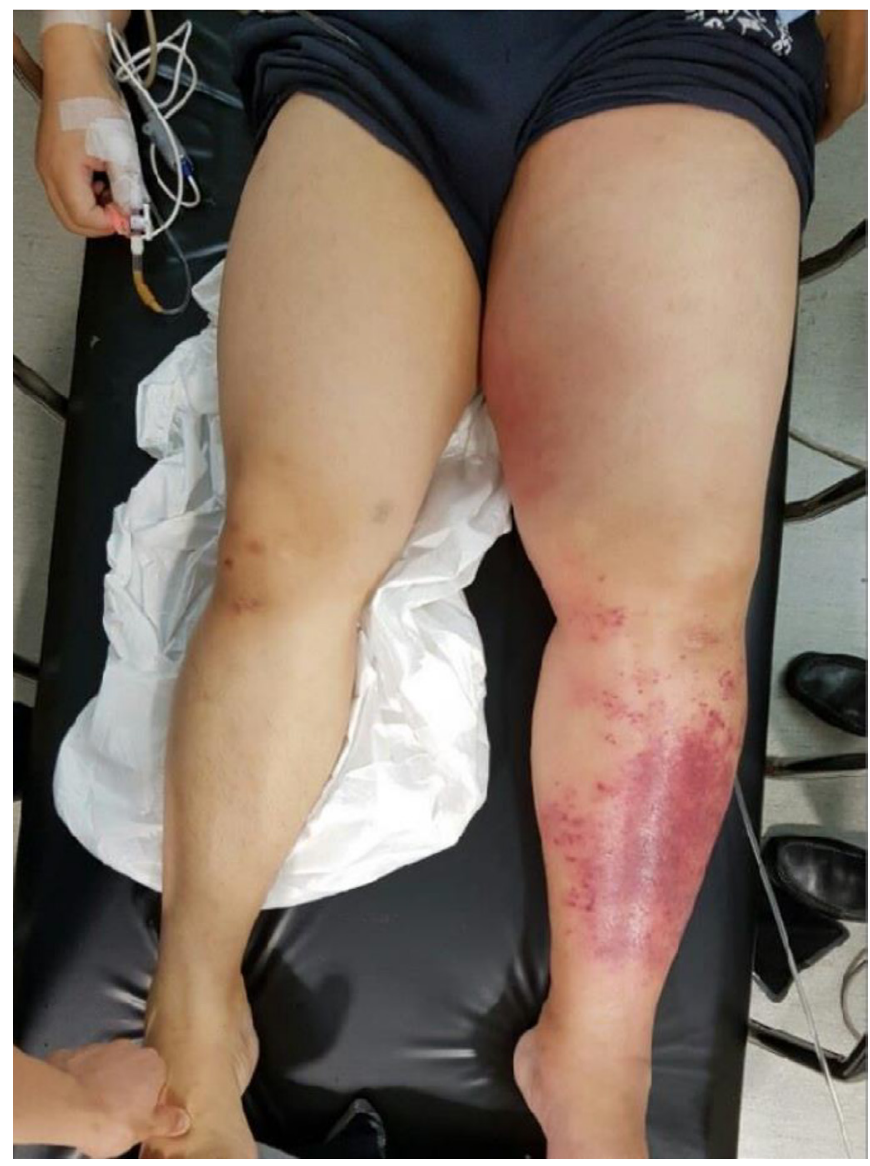

Figure. 1. Left leg edema had worsened after a traffic accident. 

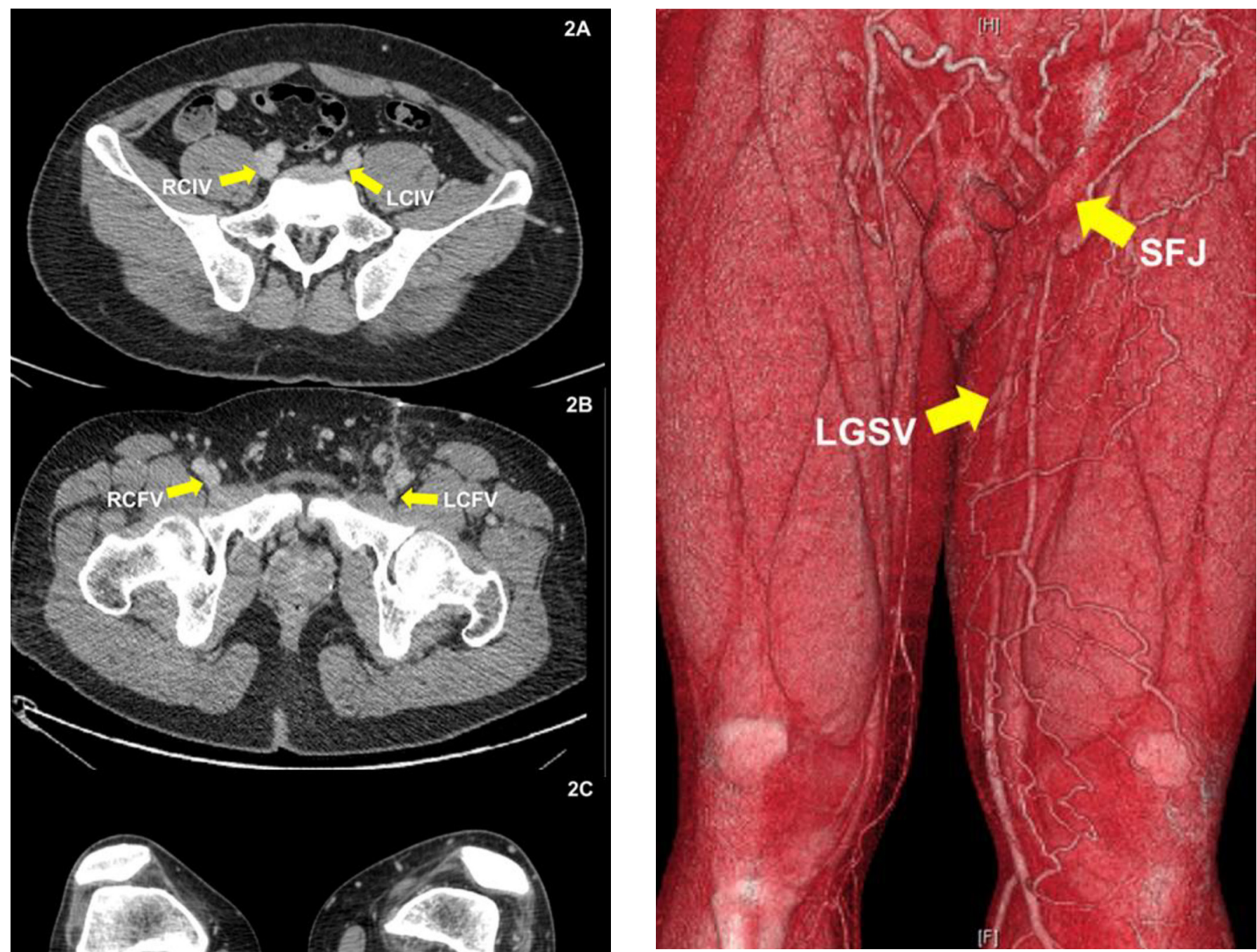

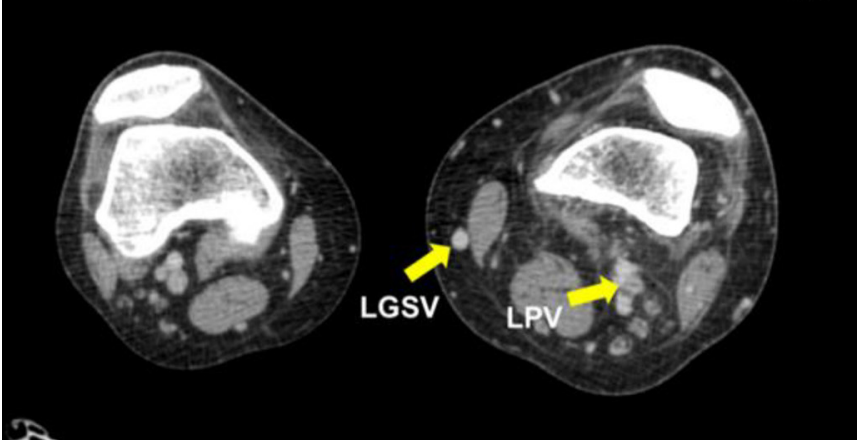

Figure. 2. Lower extremity CT scan: severely thinned LCIV, LCFV, and LPV compared to right leg veins and a huge LGSV were seen. RCIV, right common iliac vein; LCIV, left common iliac vein; RCFV, right common femoral vein; LCFV, left common femoral vein; LPV, left popliteal vein; LGSV, left great saphenous vein.

Marlborough, MA, USA) was advanced through the LIV and placed in the left superficial femoral vein (LSFV). The $0.018-$ inch guidewire was changed to 0.014 -inch guidewire. Then, for the antegrade approach, the LGSV was punctured with a micropunture needle under ultrasound guidance. The 0.014inch guidewire was passed up to the LCFV from the LGSV (Figure 4A). We injected the contrast agent through a CXI support catheter (Cook Medical, Bloomington, IN, USA) and confirmed the successful entry into the LCFV via the LGSV (Figure 4B). We captured the retrograde 0.014-inch
Figure. 3. Lower extremity CT scan (3D reconstruction image): a huge LGSV was drained into the femoral vein via the SFJ, and collateral veins were developed. LGSV, left great saphenous vein; SFJ, saphenofemoral juntion.

guidewire with the CXI support catheter (Cook Medical,) (Figure 4C). After retrograde wire externalization, an antegrade venogram showed chronic total occlusion of the LIV and the LCFV with collateral veins (Figure 4D). Balloon dilatations were done by a $4 \mathrm{~mm} \times 120 \mathrm{~mm}$ balloon (Armada 18, Abbott Vascular, Santa Clara, CA, USA) at LIV and a 3 $\mathrm{mm} \times 120 \mathrm{~mm}$ balloon (Armada 18, Abbott Vascular) at the LCFV (Figures 5A, 5B). After balloon angioplasty, venous flow was not improved (Figure 5C). Therefore, self-expandable metallic stents were deployed across the occluded segment of the vein. A $7 \mathrm{~mm} \times 40 \mathrm{~mm}$ stent (Absolute pro LL, Abbott Vascular) was inserted at LCFV and a $6 \mathrm{~mm} \times 150$ mm stent (Supera, Abbott Vascular) was inserted at the LIV (Figures 6A, 6B). Adjuvant balloon angioplasty followed. After stenting, the venous flow was significantly improved (Figure 6C), and also the patient's left leg was noticeably less swollen. 


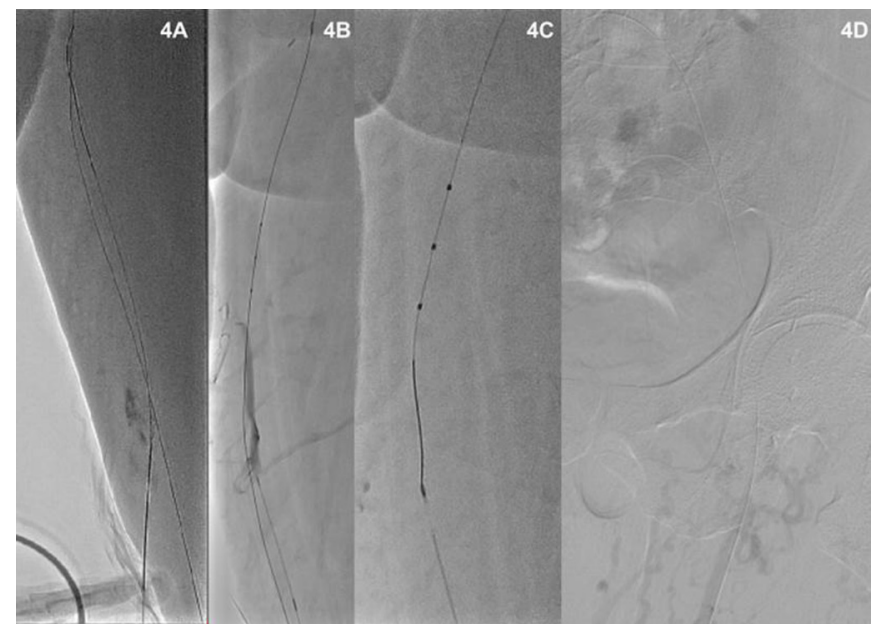

Figure. 4. A 0.014-inch guidewire was passed up to the LCFV from the LGSV (A). We confirmed the successful entry of the microcatheter into the LCFV via the LGSV by injecting contrast medium (B). We captured the retrograde 0.014-inch guidewire with a microcatheter (C). An antegrade venogram showed chronic total occlusion of the LIV and the LCFV with collateral veins (D). LCFV, left common femoral vein; LGSV, left great saphenous vein; LIV, left iliac vein.

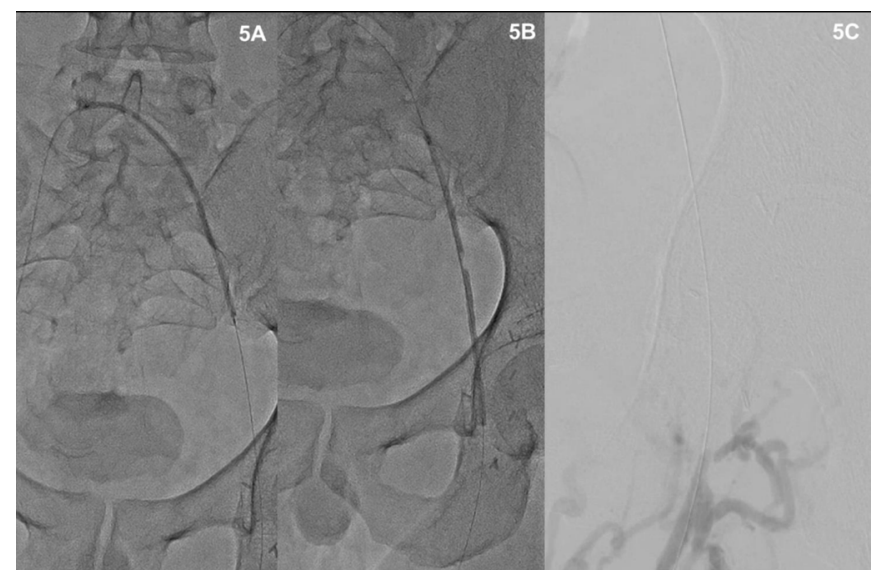

Figure. 5. Balloon dilatations were done by a $4 \mathrm{~mm} \times 120 \mathrm{~mm}$ balloon at the LIV (A) and a $3 \mathrm{~mm} \times 120 \mathrm{~mm}$ balloon at the LCFV (B). After balloon angioplasty, venous flow was not improved (C). LCFV, left common femoral vein; LIV, left iliac vein.

\section{DISCUSSION}

Chronic venous disease (CVD) is defined as "(any) morphological and functional abnormalities of the venous system of long duration manifested either by symptoms and/ or signs indicating the need for investigation and/or care" [Eklof 2009]. Patients can present with signs and symptoms ranging from mild leg pain to skin changes, venous claudication, or ulceration [Seager 2016]. Most CVD cases are caused by a nonthrombotic (primary or idiopathic) or postthrombotic (secondary) cause. Either type includes reflux, obstruction, or a combination of the two, which is most common [Raju 2009a]. Chronic obstruction of the iliac vein,

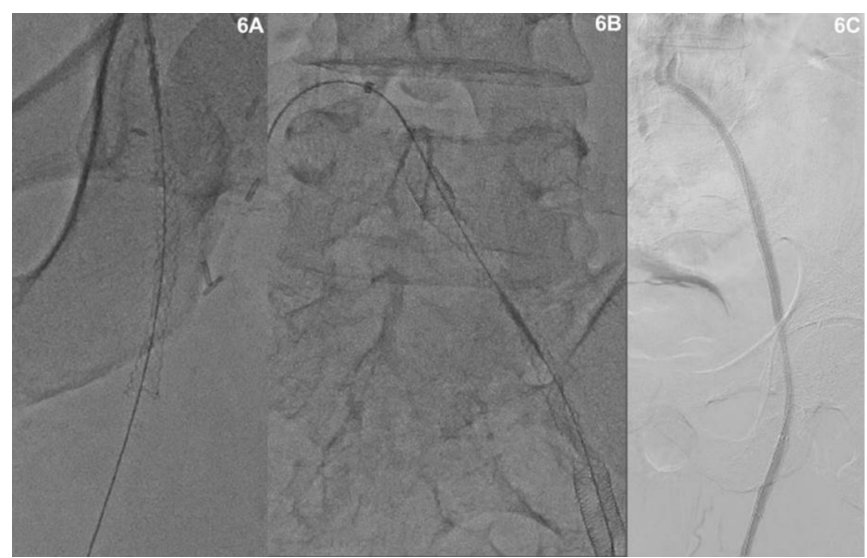

Figure. 6. A $7 \mathrm{~mm} \times 40 \mathrm{~mm}$ stent was inserted at the LCFV (A), and a $6 \mathrm{~mm} \times 150 \mathrm{~mm}$ stent was inserted at the LIV (B). Adjuvant balloon angioplasty followed. After stenting, the venous flow was significantly improved (C). LCFV, left common femoral vein; LIV, left iliac vein.

which is the common outflow tract of the lower limb, can cause severe symptoms because of insufficient compensation by a collateral vein [Neglen 2007]. Venovenous bypass has been the standard in treating chronic iliac vein obstruction [Raju 2009b, Raju 2010]. However, endovenous stenting is the current treatment of choice in chronic iliac vein obstruction [Mussa 2007]. Previous studies demonstrated the efficacy and safety of iliofemoral vein stenting for CVD in both post-thrombotic syndrome and nonthrombotic iliac vein lesions [Neglen 2007; Raju 2009b; Raju 2010; Razavi 2015; Wen-da 2016].

For iliofemoral vein stenting, antegrade venography is essential to determine the degree, length, and site of obstruction and confirm the stent dilatation and lesion coverage [Mussa 2007]. For the antegrade approach in a usual situation, low thigh access including ipsilateral femoral or popliteal vein is necessary to allow stent deployment up to and below the inguinal ligament. However, in this patient, femoral or popliteal vein access was not feasible because of thinning. Given that the GSV originates from the dorsal venous arch in the foot and travels anteriorly to the medial malleolus and ascends along the anteromedial area of the calf and into the thigh and drains into the common femoral vein via the SFJ, we decided to use the GSV as an access [Black 2014; Baliyan 2016]. Previously, small saphenous vein (SSV) and posterior tibial vein (PTV) access in catheter-directed thrombolysis of acute entire limb deep vein thrombosis has been reported [Liu 2018]. However, there has been no report of an approach through the GSV in iliofemoral vein stenting of CVD patients.

In limbs with primary nonthrombotic disease, the obstructive lesions commonly occur in both the iliac vein and femoral vein. Raju et al reported the frequent stenting of the entire iliofemoral vein segment in their study [Raju 2010]. GSV can be a good alternative for access when femoral vein obstruction is accompanied by iliac vein obstruction. GSV access has several advantages compared to conventional 
popliteal vein access. GSV access can reduce patient discomfort because the patient does not need to be in the prone position for a long time and the probability of hematoma formation due to arterial puncture is low. Liu et al reported catheter-directed thrombolysis of acute entire limb deep vein thrombosis from SSV or PTV access. In their study, SSV access showed some limitations. First, the stent delivery rod is short and requires an extra common femoral vein access to deploy the stent at the iliac vein lesion. Second, there is anatomic variation in the saphenopopliteal junction, which makes access to the deep vein area difficult via SSV [Liu 2018]. In this regard, iliofemoral vein stenting through SSV seems unsuitable.

CVD patients commonly have diffuse occlusion throughout the lower extremity vein. Therefore, it is important to plan for access by analyzing lower extremity CT scans before EVT. The GSV access is safe and feasible in stenting of chronic iliofemoral vein obstruction.

\section{CONCLUSION}

Iliofemoral vein stenting is effective and safe to treat chronic iliofemoral vein obstruction. The GSV access is safe and feasible for iliofemoral vein stenting.

\section{ACKNOWLEDGMENTS}

Funding acknowledgments: This research received no specific grant from any funding agency in the public, commercial, or not-for-profit sectors.

Conflict of interest statement: The authors declare no conflict of interest in preparing this article.

Informed consent: Informed consent has been obtained from the patient for publication of the case report and accompanying images.

\section{REFERENCES}

Baliyan V, Tajmir S, Hedgire SS, Ganguli S, Prabhakar AM. 2016. Lower extremity venous reflux. Cardiovasc Diagn Ther 6(6):533-43.

Black CM. 2014. Anatomy and physiology of the lower-extremity deep and superficial veins. Tech Vasc Interv Radiol 17(2):68-73.

Eklof B, Perrin M, Delis KT, Rutherford RB, Gloviczki P. 2009. Updated terminology of chronic venous disorders: the VEIN-TERM transatlantic interdisciplinary consensus document. J Vasc Surg 49(2):498-501.

Liu G, Liu X, Wang R, et al. 2018. Catheter-directed thrombolysis of acute entire limb deep vein thrombosis from below the knee access: a retrospective analysis of a single-center experience. Catheter Cardiovasc Interv 91(2):310-7.

Mussa FF, Peden EK, Zhou W, Lin PH, Lumsden AB, Bush RL. 2007. Iliac vein stenting for chronic venous insufficiency. Tex Heart Inst J 34(1):60-6.

Neglen P, Hollis KC, Olivier J, Raju S. 2007. Stenting of the venous outflow in chronic venous disease: long-term stent-related outcome, clinical, and hemodynamic result. J Vasc Surg 46(5):979-90.

Raju S, Neglen P. 2009. Chronic venous insufficiency and varicose veins. N Engl J Med 360(22):2319-27.

Raju S, Neglen P. 2009. Percutaneous recanalization of total occlusions of the iliac vein. J Vasc Surg 50(2):360-368.

Raju S, Darcey R, Neglen P. 2010. Unexpected major role for venous stenting in deep reflux disease. J Vasc Surg 51(2):401-8; discussion 408.

Razavi MK, Jaff MR, Miller LE. 2015. Safety and effectiveness of stent placement for iliofemoral venous outflow obstruction: systematic review and meta-analysis. Circ Cardiovasc Interv 8(10):e002772.

Seager MJ, Busuttil A, Dharmarajah B, Davies AH. 2016. Editor's Choice - a systematic review of endovenous stenting in chronic venous disease secondary to iliac vein obstruction. Eur J Vasc Endovasc Surg. 51(1):100-120.

Wen-da W, Yu Z, Yue-Xin C. 2016. Stenting for chronic obstructive venous disease: a current comprehensive meta-analysis and systematic review. Phlebology. 31(6):376-89. 